\title{
Desafíos de la Red Asistencial Pública de Chile en la Espe- cialidad de Trastornos Temporomandibulares y Dolor Orofacial
}

\author{
Challenges of the Chilean Public Health Care System Network in \\ the Temporomandibular Disorders and Orofacial Pain Specialty
}

\author{
Abarzúa P. ${ }^{1}$; Coronado L. ${ }^{2} \&$ Casassus R. ${ }^{3}$
}

\begin{abstract}
ABARZÚA, P.; CORONADO, L. \& CASASSUS, R. Desafíos de la red asistencial pública de Chile en la especialidad de trastornos temporomandibulares y dolor orofacial. Int . J. Odontostomat., 13(4):475-480, 2019.

RESUMEN: Los Trastornos Temporomandibulares (TTM) son un conjunto de patologías musculares y articulares que afectan al sistema masticatorio. Según estadísticas nacionales, la prevalencia de al menos un diagnóstico de TTM en la población adulta corresponde al 49,6 \%, mientras que el 19,6 \% padece de un dolor temporomandibular severo. La atención de pacientes afectados por estos trastornos corresponde al odontólogo especialista en trastornos temporomandibulares y dolor orofacial (TTM y DOF), especialidad reconocida legalmente desde el año 2013 por el decreto 8 del Ministerio de Salud. Estas patologías no están incorporadas a las prestaciones de salud oral de las Garantías Explícitas en Salud (GES) y recientemente se han comenzado a establecer protocolos de derivación a la especialidad desde la Atención Primaria Odontológica. En el presente estudio se realiza un análisis de la situación actual de la especialidad de TTM y DOF en la red pública de atención y se plantean posibles soluciones y mejoras a los problemas encontrados.
\end{abstract}

PALABRAS CLAVE: salud pública, prestación de asistencia sanitaria, trastornos temporomandibulares, dolor orofacial.

\section{INTRODUCCIÓN}

Los Trastornos Temporomandibulares (TTM) corresponden a un conjunto heterogéneo de patologías que afectan a los músculos masticatorios, las articulaciones temporomandibulares (ATM) y otros tejidos envueltos en el movimiento de la mandíbula (Laskin et al., 2006).

Los TTM son la causa principal de dolor maxilofacial de origen no dentario, los cuales se presentan con una sintomatología muy variable, siendo los principales el dolor muscular y/o articular, sonidos en la articulación temporomandibular (ATM), problemas de la dinámica mandibular, restricción de la apertura mandibular, además de presentarse con cefaleas y otalgias asociadas (LeResche, 1997). Estos síntomas interfieren en la capacidad de concentración, el sueño y afectan la calidad de vida de las personas (Bitiniene et al., 2018). Los TTM pueden afectar a todas las edades, pero se presentan más frecuentemen- te en la población entre 20 y 40 años (Manfredini et al., 2011). La actual prevalencia de TTM en la población general es un tema de debate, principalmente debido a las diferencias en los criterios diagnósticos de las distintas investigaciones (Manfredini et al.). Sin embargo, estudios internacionales han estimado que en la población adulta general, el 9,7\% presenta dolor muscular, $11,4 \%$ desplazamientos discales y el 2,6 $\%$ trastornos dolorosos o degenerativos articulares (Manfredini et al.). En Chile, sólo existe un estudio de prevalencia de trastornos temporomandibulares a nivel de población general, el cual fue realizado en población adulta del Servicio de Salud de Valparaíso y San Antonio, obteniendo una prevalencia de al menos un diagnóstico de TTM en un 49,6 \% y un $19,6 \%$ de con TTM severo (Guerrero et al., 2017). Estas estadísticas muestran una elevada prevalencia de TTM en la población adulta chilena, lo cual implica un desafío importante para la red asistencial odontológica de cada

\footnotetext{
${ }^{1}$ Especialista Complejo Hospitalario San José, Facultad de Medicina Clínica Alemana, Universidad del Desarrollo, Santiago, Chile.

${ }^{2}$ Magíster en Salud Pública, Universidad de La Frontera, Temuco, Chile.

${ }^{3}$ Facultad de Medicina Clínica Alemana, Universidad del Desarrollo, Santiago, Chile.
} 
Servicio de Salud (SS) y la necesidad de conocer cuál es la oferta de la especialidad de TTM y DOF en la red asistencial pública, su certificación y lista de espera (LE) respectiva.

\section{MATERIAL Y MÉTODO}

Se realizó un estudio observacional de corte transversal, con un enfoque cuantitativo de la situación de la especialidad de TTM y DOF en la red asistencial pública Chilena. Para ello, se incluyó en el estudio a los 29 Servicios de Salud (SS) de Chile. Los parámetros a medir fueron: LE de cada SS, cantidad de profesionales encargados de la LE, horas clínicas destinadas, certificación de los profesionales y la existencia de protocolos de referencia y contrarreferencia en la especialidad. El método por el cual se recabaron los datos fue a través de una solicitud directa a cada uno de los servicios de salud vía Ley de Transparencia, la cual es una ley que reconoce el derecho de todas las personas al acceso a la Información pública y que entró en vigencia en abril del 2009 (Ley 20.285). La solicitud constaba de 5 preguntas.

1) Cantidad de Lista de espera en TTM y DOF del servicio respectivo respecto al último corte

2) Nómina de los profesionales encargados de la Lista de Espera de TTM y DOF

3) Horas de atención clínica destinadas a TTM y DOF

4) Certificación de los profesionales encargados de la Lista de Espera de TTM y DOF

5) Protocolo de referencia y contrarreferencia de la especialidad de TTM y DOF.

La solicitud fue enviada a todos los servicios el día 6 de agosto del 2018, teniendo un plazo para dar respuesta hasta el día 4 de septiembre. De los 29 Servicios de salud, 17 contestaron dentro del primer plazo, los otros 12 SS dieron su respuesta en la prórroga o tras ponerse en contacto directo con los encargados de transparencia. El promedio de días de respuesta fue de 29 días, siendo la más veloz de 2 días (SS Coquimbo) y la más retrasada de 66 días (SS Metropolitano Sur). La LE declarada por los SS varían respecto a la fecha del último corte, correspondiendo la más cercana a Junio de 2018 y la más lejana a Diciembre del 2017 (SS Metropolitano Sur). La certificación de los profesionales encargados de las listas de espera de TTM y DOF fue contrastado con el registro de la Superintendencia de Salud en Noviembre del 2018. Hubo 2 SS (Metropolitano Sur
Oriente y Metropolitano Occidente) con cargos declarados en TTM y DOF que, debido a la falta de información, no se logró contrastar con la base de datos de la Superintendencia de Salud, por lo que estos fueron clasificados como "no contrastado". Esto ocurrió debido a la falta de información entregada por los SS. Se determinó la cantidad de especialistas en TTM y DOF inscritos en la Superintendencia de Salud, mediante un requerimiento ingresado a la misma institución el día 31 de Julio del 2018, obteniendo respuesta el 24 de agosto del mismo año. El mismo método se utilizó para conocer la población de usuarios inscritos en el Fondo Nacional de Salud (FONASA) por cada SS, enviando la solicitud el 28 de Noviembre del 2018 y obteniendo la respuesta el 6 de Diciembre del mismo año (una excepción fue el SS Aysén cuyo dato fue extraído desde el boletín estadístico de FONASA 2016-2017 por no encontrarse en la respuesta obtenida desde esa institución).

En la determinación del protocolo de referencia y contrarreferencia de TTM y DOF, en el caso de que este se encontrase inserto en el protocolo de otras especialidades, se consideró como "no existente".

El respaldo de las respuestas de los SS y la superintendencia fueron almacenados para futuras consultas. Los datos fueron agrupados y analizados en una planilla $\mathrm{MS}^{-E_{x c e}{ }^{\circ}} 2010$.

\section{RESULTADOS}

Del total de 29 Servicios de Salud en Chile, 6 Servicios manifiestan no tener lista de espera debido a no contar con especialista, a no tener abierta la cartera de prestaciones o por considerarla como brecha. EI SS Atacama manifiesta tener LE a pesar de no tener especialista. La sumatoria de LE en TTM y DOF de los SS que declaran tenerla, es de 15.040 usuarios. La LE de cada SS está representada en el gráfico anexo (Fig. 1). En el caso del SS Metropolitano Suroriente, la LE de TTM y DOF está contenida con la LE de periodoncia, siendo aproximadamente un $25 \%$ de la misma.

De los 109 especialistas en TTM y DOF que están inscritos en la Superintendencia de Salud, 22 tienen algún cargo en un SS, lo que representa el 20 $\%$ del total de especialistas. existen un total de 33 cargos para TTM y DOF en Chile (Tabla I), de estos el $66,7 \%$ corresponde a profesionales con certificación 
en TTM DOF, habiendo un $27,3 \%$ que no tiene certificación en la especialidad o que se encuentran certificados en otra especialidad debido a la falta de información. Hubo dos casos no fueron posible contrastar en la Superintendencia de Salud. Respecto a los cargos cada profesional, el 48,5\% (15 cargos) de estos corresponden a puestos de 11 horas o menos (ya que en el caso del SS Aysén depende de la "necesidad"), $27,3 \%$ a 22 h ( 9 cargos) , $6 \%$ corresponden de $33 \mathrm{~h}$ ( 2 cargos) y un $18,2 \%$ a $44 \mathrm{~h}$ (6 cargos). El promedio nacional de horas contratadas en la especialidad es de $23 \mathrm{~h}$ con un promedio de 1,1 cargos por SS.
Los 15 SS que cuentan solo con profesionales certificados, representan el $53 \%$ de la totalidad de población FONASA. Aquellos que no cuentan con ningún tipo de oferta en TTM y DOF (6 SS) corresponden al 13 $\%$ de usuarios FONASA (correspondiente al Servicio de Salud Maule el 53,6 \% del total de esta falencia). Los 4 SS que no tienen profesionales certificados en la especialidad representan $8 \%$ del global de FONASA, los 2 SS en los cuales no se pudo contrastar sus profesionales al abarcan al $16 \%$ y los 2 SS restantes, los cuales contaban con equipos compuestos por profesionales certificados y sin certificación en TTM y DOF constituyen el $10 \%$ de la población usuaria de FONASA (Fig. 2).

\section{LISTA DE ESPERA TTM Y DOF EN LOS SERVICIOS DE SALUD}

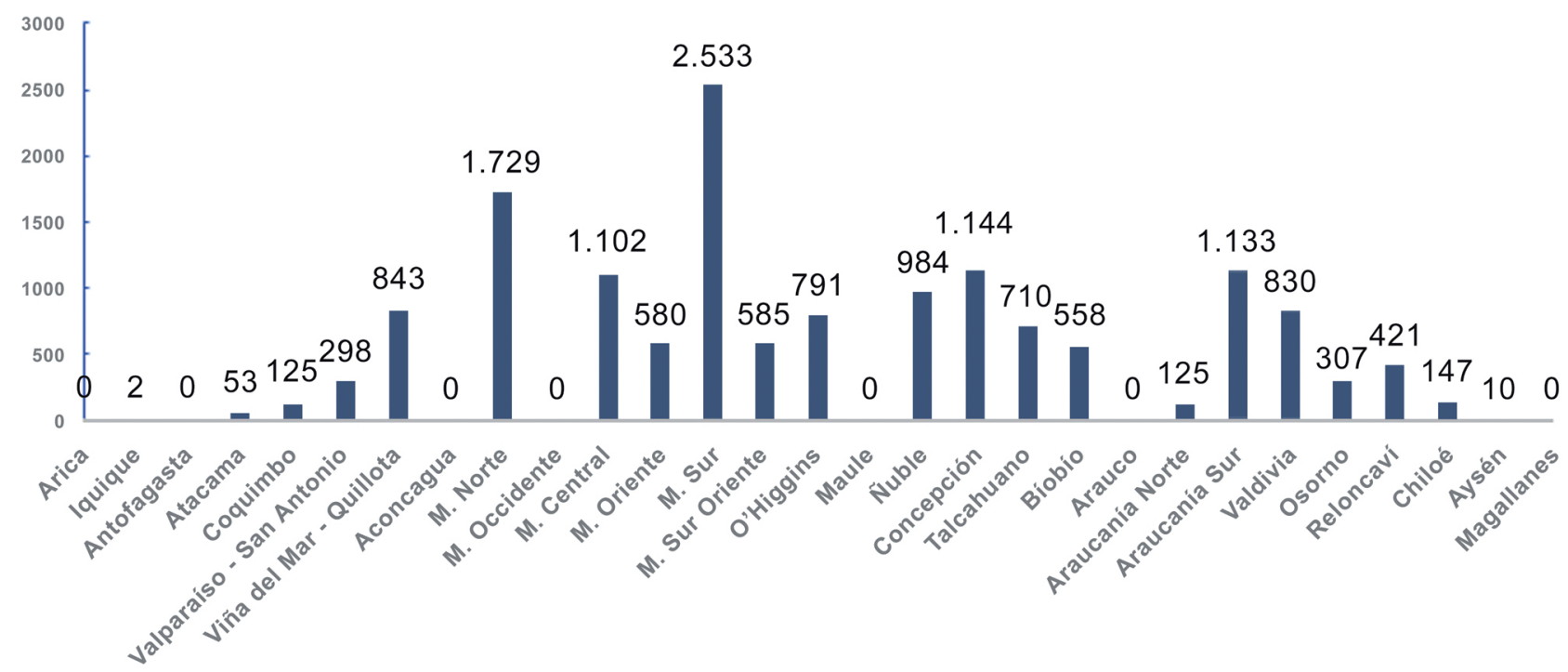

Fig. 1. Lista de Espera de TTM y DOF en los Servicios de Salud de Chile. * LE de TTM y DOF contenida en LE de Periodoncia.

TIPO DE OFERTA DE TTM Y DOF EN LA POBLACIÓN FONASA

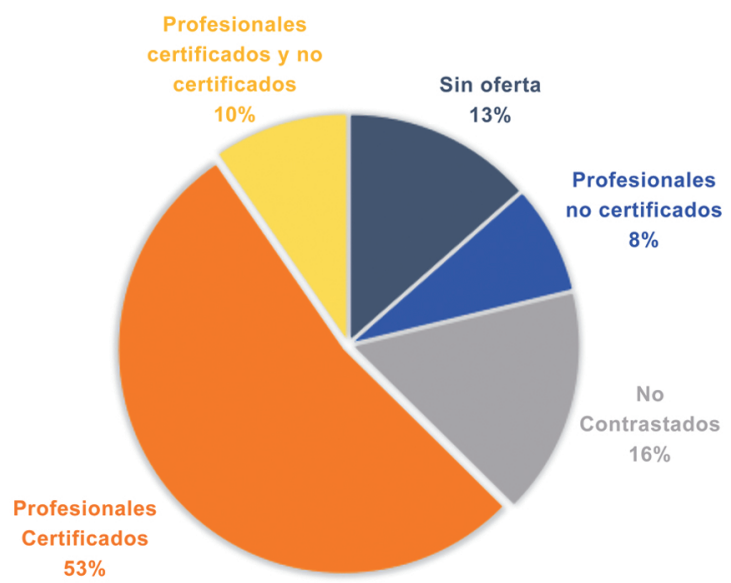

Fig. 2. Tipo de oferta en TTM y DOF en la población FONASA.
Un $44,8 \%$ de los SS cuenta con un protocolo de referencia/contrarreferencia en TTM y DOF propiamente tal (ver Tabla I).

\section{DISCUSIÓN}

La VII Reunión Regional de los Observatorios de Recursos Humanos en Salud, realizada en Toronto en 2005, determinó que el período 2006-2015 se constituye en "Una Década de Recursos Humanos en Salud". Tal determinación es debida a la necesidad de enfrentar los serios desafíos con relación al desarrollo de esos recursos en la región de las Américas (Organización Panamericana de la Salud, 2012). En Chile desde el año 2002, el país implementa una reforma sanitaria orientada a definir garantías explícitas en 
Tabla I. Número de cargos y cargos con certificación, horas de especialidad, lista de espera, población, presencia de protocolos de derivación en TTM y DOF por servicio de salud.

\begin{tabular}{|c|c|c|c|c|c|c|}
\hline SERVICIO DE SALUD & $\begin{array}{c}\mathbf{N}^{\mathbf{a}} \\
\text { CARGOS }\end{array}$ & $\begin{array}{c}N^{\circ} \text { CARGOS } \\
\text { CERTIFICADOS }\end{array}$ & $\begin{array}{l}\text { HORAS } \\
\text { TOTALES }\end{array}$ & $\begin{array}{l}\text { POBLACIÓN } \\
\text { INSCRITA }\end{array}$ & $\begin{array}{l}\text { LE TTM y } \\
\text { DOF }\end{array}$ & PROTOCOLC \\
\hline Arica & - & - & - & 186.409 & No & No \\
\hline Iquique & 1 & 1 & 22 & 275.952 & 2 & No \\
\hline Antofagasta & 1 & 1 & 22 & 348.643 & No & $\mathrm{Si}$ \\
\hline Atacama & - & - & - & 226.677 & 53 & No \\
\hline Coquimbo & 1 & 1 & 44 & 609.989 & 125 & No \\
\hline Valparaíso - San Antonio & 1 & 1 & 44 & 366.480 & 298 & No \\
\hline Viña del Mar - Quillota & 2 & 2 & 55 & 771.027 & 843 & $\mathrm{Si}$ \\
\hline Aconcagua & - & - & - & 166.960 & No & No \\
\hline M. Norte & 1 & 1 & 44 & 652.355 & 1.729 & $\mathrm{Si}$ \\
\hline M. Occidente & 1 & NC & 22 & 1.013 .871 & Brecha & No \\
\hline M. Central & 2 & 2 & 33 & 279.778 & 1.102 & No \\
\hline M. Oriente & 3 & 2 & 33 & 613.447 & 580 & No \\
\hline M. Sur & 1 & 1 & 44 & 1.020 .677 & 2.533 & $\mathrm{Si}$ \\
\hline M. Sur Oriente & 2 & $0\left(N C^{*}\right)$ & 53 & 1.032 .016 & 585 & $\mathrm{No}^{* *}$ \\
\hline O’Higgins & 1 & 1 & 22 & 704.684 & 791 & No \\
\hline Maule & - & - & - & 911.265 & No & No \\
\hline Ñuble & 1 & 0 & 33 & 350.066 & 984 & $\mathrm{Si}$ \\
\hline Concepción & 1 & 1 & 22 & 433.587 & 1.144 & $\mathrm{Si}$ \\
\hline Talcahuano & 1 & 1 & 22 & 298.886 & 710 & $\mathrm{Si}$ \\
\hline Biobío & 2 & 2 & 22 & 278.678 & 558 & $\mathrm{Si}$ \\
\hline Arauco & - & - & - & 93.963 & No & No \\
\hline Araucanía Norte & 1 & 1 & 11 & 151.662 & 125 & No \\
\hline Araucanía Sur & 3 & 2 & 33 & 600.696 & 1.133 & $\mathrm{Si}$ \\
\hline Valdivia & 1 & 0 & 11 & 317.516 & 830 & $\mathrm{Si}$ \\
\hline Osorno & 1 & 0 & 11 & 210.413 & 307 & $\mathrm{Si}$ \\
\hline Reloncaví & 1 & 1 & 44 & 313.910 & 421 & $\mathrm{Si}$ \\
\hline Chiloé & 1 & 1 & 22 & 166.374 & 147 & $\mathrm{Si}$ \\
\hline Aysén & 3 & 0 & Necesidad & 85.091 & 10 & No \\
\hline Magallanes & - & - & - & 114.990 & Brecha & No \\
\hline
\end{tabular}

LE: lista de espera, NC: no contrastado, *un caso sin contrastar, ${ }^{* *}$ contenido en protocolo de periodoncia.

salud exigibles para los ciudadanos y mejorar los modelos de atención y gestión del sistema (Méndez, 2009). El impacto de las reformas y políticas sanitarias requiere el apoyo crucial de equipos de salud bien conformados, capacitados, motivados y completos (Homedes \& Ugalde, 2005), siendo entonces, el recurso humano el área a desarrollar si queremos un sistema de salud que responda eficazmente a las necesidades sanitarias de la población. Este sistema de salud debe poseer un enfoque estratégico que implica la presencia de una cartera de prestaciones y especialidades ajustada a las demandas de salud de la población lo cual es fundamental para el desarrollo estratégico de la red asistencial. La creación del recurso humano en salud y su funcionamiento en red, debe basarse en la evidencia científica epidemiológica disponible lo que es un insumo necesario para la estimación de brechas de especialidades médicas y odontológicas (Méndez \& Torres, 2010).
La LE en TTM y DOF en Chile se genera por varias causas, entre ellas por que el sistema tiene brechas de recursos humanos, financieros y de equipos y de infraestructura (Alarcón Rojas et al., 2018). Se debe tener en cuenta que la mayoría de las patologías atingentes a la especialidad de TTM y DOF son de carácter doloroso, por lo que es necesario pesquisar y tratar tempranamente estas patologías desde atención primaria para así dar un tratamiento oportuno o ser referido tempranamente (Ghurye \& McMillan, 2017). Las condiciones más comunes de dolor orofacial crónico se relacionan con un mal manejo del dolor agudo recurrente dental o de un trastorno temporomandibular (Renton, 2017). Un correcto y temprano tratamiento es fundamental para evitar una cronificación del dolor, lo que deteriora la calidad de vida de las personas y que al mismo tiempo complejiza el diagnóstico y tratamiento de la patología (Renton). Sumado a esto, hay que tener en cuenta el impacto 
económico del dolor crónico, el cual tiene un importante componente social asociado a la disminución de la productividad (Phillips, 2009). Por lo que un mejor manejo del dolor crónico reducirá la discapacidad y aumentará la calidad de vida los pacientes, también reducirá los costos para el sistema de salud y los importantes costos sociales para las personas (Espinoza et al., 2017).

Con el objetivo de dar una correcta y oportuna respuesta en el campo de los TTM y DOF, hace falta mejorar los servicios odontológicos en las redes integradas, fortalecer el desarrollo del recurso humano odontológico competente en los distintos niveles de la red y aumentar la dotación de recurso humano odontológico (Subsecretaría de Salud Pública, 2017, 2108). En Chile existen 33 cargos de especialista en TTM y DOF con un promedio de $23 \mathrm{~h}$ semanales, esto sin duda es un avance con respecto a lo reportado en el Plan de Salud Bucal 2018-2030 del Ministerio de Salud (Subsecretaría de Salud Pública, 2017) en el cual se reportaban 25 cargos, sin embargo sigue siendo insuficiente considerando que casi la mitad de la población usuaria de FONASA no puede acceder a una atención de calidad dada por un especialista en el área. Por lo anterior, es fundamental el aumento de la cantidad de horas especialista en TTM y DOF en los distintos niveles de la red asistencial de los Servicios de Salud, las cuales actualmente son escasas. Además, es necesaria la capacitación de los odontólogos generales y demás profesionales de la salud, labor que sin duda debe recaer en una primera instancia en los especialistas en TTM y DOF, pero también en los mismos odontólogos generales una vez capacitados en el diagnóstico del área (Mejersjö et al., 2018). Sumado a esta última idea y considerando la alta prevalencia de TTM en la población, sería beneficioso fortalecer la formación desde pregrado, destinando mayor cantidad de horas teóricas, preclínicas y clínicas dirigido a las patologías más prevalentes de la articulación temporomandibular y músculos masticatorios (Nordin et al., 2016).

Las cifras de las LE son dinámicas en el tiempo y se utilizan para propósitos importantes como medir el rendimiento, para planificación local y para proporcionar información a los pacientes sobre el acceso a cuidado de la salud (Godden \& Pollock, 2009). Las diferencias en la recopilación de datos y los métodos tienen una importante impacto en las estadísticas publicadas, en su calidad y sus resultados, determinando finalmente quién aparece y quién no en la LE (Godden \& Pollock). Esto hace difícil interpretar las estadísticas de espera en relación a la necesidad del acceso a la atención de salud, porque además generalmente no cuentan con información relativa a la prontitud del tratamiento, como es la severidad de la patología o condición del afectado (Godden \& Pollock). La inexistencia de LE en algunos SS, más bien habla de una lista de espera escondida o no transparentada, por lo cual esta situación no significa una ausencia de la necesidad de tratamiento ni de estas patologías en la población. Al no haber LE es los SS, junto a la imposibilidad de generar una LE y sumado al hecho de no contar con estudios de prevalencia en esas poblaciones específicas, ¿como podemos reflejar la necesidad de incorporación de un especialista en TTM y DOF?. Quizás la respuesta puede estar en aquellos SS en que hasta hace unos años no contaban ni con LE ni con especialista en el área. Un ejemplo de ellos es el SS Coquimbo, el cual hasta hace no más de 3 años incorporó a un especialista en TTM y DOF, y a la fecha ya tienen 125 usuarios en LE. Esta falta de reflejo sobre la necesidad de la especialidad también puede verse en los casos de SS en que los pacientes de TTM y DOF son derivados a otras especialidades o donde son atendidos por profesionales sin certificación en la especialidad. Cabe señalar que solo son 15 los SS que cuentan con especialistas certificados en TTM, representando el $53 \%$ de la población que utiliza el servicio de salud pública, por lo que solo la la mitad de los usuarios FONASA pueden acceder a una atención de calidad con un especialista certificado.

Por otro lado, un problema relevante es la ausencia de un protocolo de referencia y contrarreferencia en más de la mitad de los servicios. Los protocolos son fundamentales para mejorar la capacidad resolutiva del nivel primario de atención, mejorando la pertinencia y acceso a la atención de especialidades odontológicas, fortaleciendo a los equipos de los distintos niveles en la entrega de servicios integrales, mejorando el trabajo en red y dando una atención más oportuna a los usuarios (Subsecretaría de Salud Pública, 2017, 2018).

La evidencia disponible indica que existe una alta prevalencia de TTM en la población adulta chilena $(49,63 \%)$, lo que sumado a la falta de cobertura de atención especializada en TTM y DOF, se constituye una brecha sanitaria emergente y la necesidad de conformación de equipos de especialidad odontológica en establecimientos de atención secundaria y terciaria. La red asistencial pública chilena muestra una baja cobertura en la especialidad de TTM y DOF, lo cual se demuestra en la ausencia de algún especialista certi- 
ficado en al menos 5 servicios de salud y en la ausencia total de profesionales encargados de este problema de salud en otros 6 servicios; en la ausencia de protocolos en más de la mitad de SS y en la reducida cantidad de horas de especialista en la red pública de salud. Una adecuada medición y cumplimiento de las directrices de trabajo planteadas en el Plan de Nacional de Salud Bucal 2018-2030 (Subsecretaría de Salud Pública, 2017) deberían ser beneficiosas para mejorar en parte la situación, pero esta problemática demanda también un esfuerzo desde los mismos profesionales que conforman la red asistencial.

Todo lo anterior representa una brecha sanitaria presente en la red asistencial odontológica que debe ser abordada y solucionada adecuadamente por las autoridades pertinentes con el fin dar cobertura, calidad y acceso a la especialidad de TTM y DOF en todos los niveles de la red del sistema de salud público Chileno.

ABARZÚA, P.; CORONADO, L. \& CASASSUS, R. Challenges of the Chilean public health care system network in temporomandibular disorders and orofacial pain specialty. Int. J. Odontostomat., 13(4):475-480, 2019.

ABSTRACT: Temporomandibular Disorders (TMD) comprise a heterogenous group of muscle and joint disorders that affect the masticatory system. According to national statistics, the prevalence of at least one diagnosis of TMD in the adult population is $49.6 \%$, while $19.6 \%$ suffers from severe temporomandibular pain. Care of patients affected by these disorders corresponds to the dentist specialized in temporomandibular disorders and orofacial pain (TMD and OFP); these particular disorders and the specialty were incorporated by the Ministry of Health in 2013.. The pathologies are not incorporated in the Explicit Health Care Guarantees (GES) and only recently protocols for a referral program were established for Primary Dental Care in this area. In this study, the present situation of the specialty of TMD and OFP in the public health care network is analyzed, as well as possible solutions and improvements to the difficulties encountered therein.

KEY WORDS: public health, health care allocation , temporomandibular disorders, orofacial pain

\section{REFERENCIAS BIBLIOGRÁFICAS}

Bitiniene, D.; Zamaliauskiene, R.; Kubilius, R.; Leketas, M.; Gailius, T. \& Smirnovaite, K. Quality of life in patients with temporomandibular disorders. A systematic review. Stomatologija, 20(1):3-9, 2018.

Espinoza, M.; Repetto, P.; Cabieses, B.; Vargas, C. \& Zitko, P. Capitulo I. Propuesta de Política Pública para el Manejo del Dolor Crónico Musculoesquelético en Chile, 2017. En: Irarrázaval, I.; Piña, E.; Letelier, M. \& Jeldes, M. I. (Eds.). Propuestas para Chile. Concurso Políticas Públicas 2017. Santiago de Chile, Pontificia Universidad
Católica de Chile, 2018. pp.19-42. Disponible: https:// politicaspublicas.uc.cl/wp-content/uploads/2018/03/CAP-I-Propuestas-para-Chile-2017.pdf

Ghurye, S. \& McMillan, R. Orofacial pain - an update on diagnosis and management. Br. Dent. J., 223(9):639-47, 2017.

Godden, S. \& Pollock, A. M. Waiting list and waiting time statistics in Britain: a critical review. Public Health, 123(1):47-51, 2009.

Guerrero, L.; Coronado, L.; Maulén, M.; Meeder, W.; Henríquez, C. \& Lovera, M. Prevalencia de trastornos temporomandibulares en la población adulta beneficiaria de Atención Primaria en Salud del Servicio de Salud Valparaíso, San Antonio. Av. Odontoestomatol., 33(3):113-20, 2017

Homedes, N. \& Ugalde, A. Human resources: the Cinderella of health sector reform in Latin America. Hum. Resour. Health, 3(1):1, 2005.

Laskin, D. M.; Greene, C. S. \& Hylander, W. L. Temporomandibular Disorders. An Evidence-Based Approach to Diagnosis and Treatment. Chicago, Quintessence Pub., 2006.

LeResche, L. Epidemiology of temporomandibular disorders: implications for the investigation of etiologic factors. Crit. Rev. Oral. Biol. Med., 8(3):291-305, 1997.

Manfredini, D.; Guarda-Nardini, L.; Winocur, E.; Piccotti, F.; Ahlberg, J. \& Lobbezoo, F. Research diagnostic criteria for temporomandibular disorders: a systematic review of axis I epidemiologic findings. Oral Surg. Oral Med. Oral Pathol. Oral Radiol. Endod., 112(4):453-62, 2011.

Mejersjö, C.; Bertilsson, O. \& Bäck, K. Short clinical examination for temporomandibular symptoms in general practice. Acta Odontol. Scand., 76(3):183-7, 2018

Méndez, C. A. \& Torres, A. M. C. Autonomía en la gestión hospitalaria en Chile: los desafíos para el recurso humano en salud. Rev. Saúde Pública, 44(2):366-71, 2010.

Méndez, C. A. Los recursos humanos de salud en Chile: el desafío pendiente de la reforma. Rev. Panam. Salud Pública, 26(3):276-80, 2009.

Nordin, S.; Dawson, A. \& Ekberg, E. C. Achieved Competencies and Satisfaction in Temporomandibular Disorders and Orofacial Pain Education. J. Oral Facial Pain Headache, 30(2):156-64, 2016.

Organización Panamericana de la Salud (OPS). Estrategia de Recursos Humanos para la Salud Universal. Observatorio Regional de Recursos Humanos de Salud. Washington D. C., Organización Panamericana de la Salud (OPS), 2012. Disponible en: https:// www.observatoriorh.org/node/907

Phillips, C. J. The cost and burden of chronic pain. Rev. Pain, 3(1):2-5, 2009.

Renton, T. Chronic orofacial pain. Oral Dis., 23(5):566-71, 2017.

Subsecretaría de Redes Asistenciales. Plan Nacional de Tiempos de Espera No GES en Chile en Modelo de Atención en RISS (Redes Integradas de Servicios de Salud) 2014-2018. Santiago de Chile, Subsecretaría de Redes Asistenciales, Ministerio de Salud, Gobierno de Chile, 2018. Disponible en: https://www.minsal.cl/wp-content/ uploads/2018/03/Plan-nacional-de-tiempos-de-espera-No-GES.pdf

Subsecretaría de Salud Pública. Plan Nacional de Salud Bucal 20182030. Santiago de Chile, Subsecretaría de Salud Pública, Ministerio de Salud, Gobierno de Chile, 2017. Disponible en: https:// www.minsal.cl/wp-content/uploads/2017/12/Plan-Nacional-Salud-Bucal-2018-2030-Consulta-P\%C3\%BAblica-20_12_2017.pdf

Dirección para correspondencia:

Pablo Abarzúa Cofré

Universidad del Desarrollo

Santiago

CHILE

Email: pabarco@gmail.com

Lcoron@gmail.com

Recibido : 16-05-2019

Aceptado: 01-07-2019 